
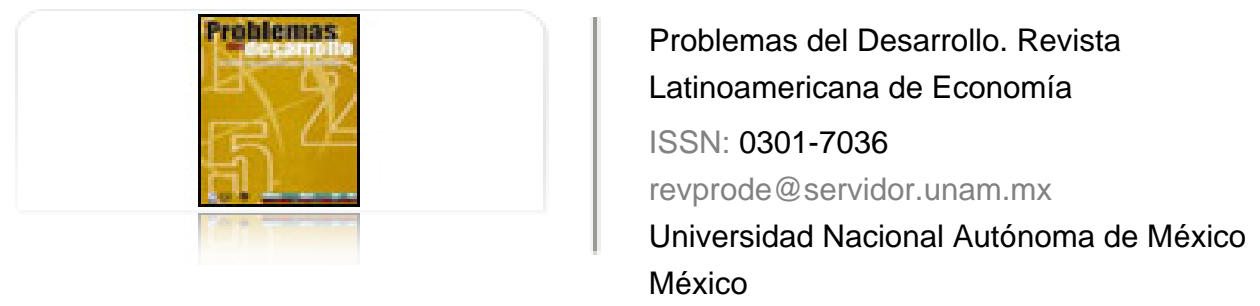

Paz Antolín, Ma. José

Efectos de la expansión de empresas transnacionales en el sector eléctrico en Guatemala Problemas del Desarrollo. Revista Latinoamericana de Economía, vol. 35, núm. 137, 2004, pp. 135-

159

Universidad Nacional Autónoma de México

Distrito Federal, México

Disponible en: http://www.redalyc.org/articulo.oa?id=11825947009

Cómo citar el artículo

- Número completo

- Más información del artículo

Página de la revista en redalyc.org

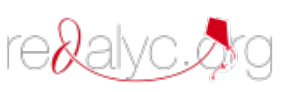

Sistema de Información Científica

Red de Revistas Científicas de América Latina, el Caribe, España y Portugal Proyecto académico sin fines de lucro, desarrollado bajo la iniciativa de acceso abierto 


\title{
EFECTOS DE LA EXPANSIÓN DE EMPRESAS TRANSNACIONALES EN EL SECTOR ELÉCTRICO EN GUATEMALA
}

\author{
Ma. José Paz Antolín*
}

Fecha de recepción: 9 de junio de 2003. Fecha de aceptación: 9 de junio de 2004.

\section{Resumen}

Durante las dos últimas décadas, varios países de América Latina llevaron a cabo la privatización y liberalización de sus sectores de servicios, entre ellos, el eléctrico. Guatemala no ha quedado al margen de este proceso y, como resultado del mismo, han llegado al país nuevas inversiones - empresas estadounidenses en el área de generación y españolas en el de distribución-. Los objetivos declarados de este procedimiento hacían referencia a una reducción del coste de la energía, una mejora en la prestación del servicio y una extensión del mismo hacia el área rural. El presente trabajo tiene como fin analizar en qué medida se han cumplido cada uno de los citados objetivos.

Palabras clave: empresas transnacionales, electricidad, Guatemala.

\begin{abstract}
During the last two decades, several countries in Latin America carried forward privatization and liberalization programs in the service sectors, including the electricity sector. Guatemala has not been left on the edge of this process and, as a result of it, new investments have flowed into the country - U.S. companies in the area of generation and Spanish companies in distribution. The declared aims of this process made reference to a reduction in the cost of energy, an improvement in the service and an extension of it into the rural areas. This study aims at analyzing to what extent each of the cited objectives has been fulfilled.
\end{abstract}

Key words: transnational corporations, electricity, Guatemala, privatization and liberalization.

* Investigadora del Departamento de Economía Aplicada I, Universidad Complutense de Madrid. Correo electrónico: ecap1w0@sis.ucm.es 


\section{Résumé}

Pendant les deux dernières décades, plusieurs pays d'Amérique Latine ont mis en place la privatisation et la libéralisation de leurs secteurs de services, parmi eux, le secteur électrique. Le Guatemala n'est pas resté à l'écart de ce processus ce qui s'est traduit par l'arrivée au pays de nouveaux investissements - entreprises américaines dans le domaine de la génération et espagnoles dans celui de la distribution-. Les objectifs déclarés dans ces processus faisaient référence à une réduction du coût de l'énergie, à une amélioration dans la prestation du service et a une extension de ce dernier vers la zone rurale. Ce travail a pour but d'analyser dans quelle mesure on a atteint chacun desdits objectifs.

Mots clés: entreprises transnationales, électricité, Guatemala, privatisation et libéralisation.

\section{Resumo}

Vários países da América Latina, durante as duas últimas décadas, realizaram a privatização e a liberalização de seus setores de serviços, entre eles o elétrico. A Guatemala não ficou à margem deste processo e, como resultado do mesmo, novos investimentos têm chegado ao país - empresas norte-americanas na área de geração e espanholas no setor de distribuição-. Os objetivos declarados destes procedimentos faziam referência a possíveis reduções do custo da energia, melhorias na prestação de serviço e aumento da oferta energética nas áreas rurais. $O$ presente trabalho se propõe a analisar de que forma foram cumpridos cada um dos citados objetivos.

Palavras-chave: empresas multinacionais, eletricidade, Guatemala, privatização e liberalização. 


\section{Introducción}

$\mathrm{H}$

asta la década de los años ochenta, el sector eléctrico en la mayoría de los países del mundo se encontraba en manos de empresas públicas, muchas de ellas integra das verticalmente y que ejercían sus actividades en situaciones de monopolio. Desde finales de los años setenta esta situación comenzó a cambiar mediante la aplicación de una serie de medidas encaminadas a liberalizar el sector y privatizar dichas empresas públicas existentes. América Latina y, particularmente Guatemala, no es ajena a este proceso. Estas políticas favorecen la expansión internacional de las empresas eléctricas, especialmente las estadounidenses y europeas, que pasan a ocupar un papel fundamental en los respectivos sectores de la mayoría de las economías latinoamericanas. Al menos desde un punto de vista formal, la implantación de estas políticas responde a la búsqueda de determinadas metas relacionadas con un aumento de la eficiencia que permita una reducción de las tarifas, una mejora de la calidad del servicio y una extensión de su cobertura a toda la población. El propósito de este trabajo es analizar las características de este proceso en el caso guatemalteco y el grado de cumplimiento de los objetivos declarados. A pesar de las particularidades propias de este caso, en muchos sentidos la situación guatemalteca es similar a la de otros países de la región.

\section{Características del modo de expansión de empresas transnacionales en el sector eléctrico en Guatemala}

En trabajos precedentes (Paz Antolín, 2002) hemos sostenido que los efectos de la llegada de inversión extranjera directa (IED) y empresas transnacionales (ET) a una economía dependen de la interrelación existente entre las ventajas competitivas de las empresas y las de localización de los países. ${ }^{1}$ En función de cómo se dé esta interrelación, la entrada de capital extranjero se plasmará en unas estrategias y modalidades concretas adoptadas por las ET, que junto a las características particulares de cada economía, condicionan la existencia de unos resultados u otros en cada país y sector. ${ }^{2} \mathrm{El}$ análisis de la industria eléctrica

1 Entendemos por ventajas de localización todos aquellos factores tanto económicos como políticos o sociales que constituyen un atractivo o un determinante para la localización de inversiones extranjeras.

2 De forma muy sintética pero clara, se expone aquí el argumento teórico que sustenta la estructura del artículo: que los efectos de la llegada de ET están en función de las estrategias, modalidades y factores de localización que operan en cada caso. Este planteamiento surge de la crítica a los argumentos maniqueos y generalistas tanto teóricos como políticos a favor o en contra de la IED y las ЕT. Por el contrario se considera que el estudio de los efectos debe realizarse a partir de las 
guatemalteca se va a fundamentar en este planteamiento. Por ello, en el presente apartado se detallan las características particulares que está adoptando el proceso de expansión de ET en dicha nación y sector para, a continuación, analizar y comprender sus efectos.

A manera de introducción: incluimos un cuadro resumen de la estructura y los principales agentes que integran el mercado eléctrico guatemalteco.

Tabla 1

Estructura del mercado eléctrico guatemalteco

\begin{tabular}{lc}
\hline $\begin{array}{l}\text { Autoridad legal } \\
\text { Autoridad regulatoria }\end{array}$ & Ministerio de Energía y Minas (MEM) \\
Operador de mercado & Comisión Nacional de Energía Eléctrica (CNEE) \\
Empresas generadoras & Administrador del Mercado Mayorista (AMM) \\
& - Empresa pública: empresa generadora de energía eléctrica (INDE) \\
& - Empresas privadas: \\
& i) Transnacionales: GGG, Enron, Tampa y TECo \\
& ii) Nacionales: ingenios azucareros y minihidráulicas \\
Empresa transportista & Empresa de transportes y control de energía eléctrica (INDE) \\
Empresas distribuidoras & Empresas privadas: \\
& - Transnacionales: EEGSA (consorcio Iberdrola), DEOCSA y DEORSA \\
& (Unión Fenosa) \\
Empresas comercializadoras & Empresas privadas: Comegsa (EEGSA), Poliwatt (Enron), Cecsa, \\
Elecno, Jacsa, Mel & Industrias y agrupaciones de consumidores con demandas por encima \\
de $100 \mathrm{kv}$
\end{tabular}

Fuente: elaboración propia con base en información del AMM y la CNEE.

\section{Factores de localización}

El proceso de liberalización y privatización constituye el principal factor de localización de ET en el sector eléctrico guatemalteco. A ello se añaden los ritmos de crecimiento de la demanda experimentados en los últimos años. Aunque es similar a lo acontecido en otros países de la región, el caso de Guatemala presenta particularidades que a continuación mencionamos. Al margen de que el marco regulatorio y la estructura del sector resultante de la reforma tenga diferencias con respecto a otros países, tres factores han sido especialmente importantes como condicionantes de las estrategias adoptadas por las ET en Guatemala: el tamaño del mercado, la situación del sector previa a la reforma y la falta de recursos energéticos como el gas. En cuanto al primero, se caracteriza por ser reducido. A pesar de que la demanda de energía eléctrica creció a un ritmo significativo, el nivel

variables señaladas. Dar una explicación exhaustiva no constituye el objetivo de este artículo que utiliza la hipótesis como punto de partida, de ahí que no se desarrolle más en el texto y que se cite otro trabajo donde el tema está abordado con mayor detalle. 
medio de consumo por habitante en Guatemala sigue siendo bajo (372kwh/habitante en 1999), ${ }^{3}$ en gran parte motivado por la alta participación de población rural. A esto hay que añadir que se trata de un país pequeño, tanto en términos de población, como de PIB. Dado el tamaño de dicho mercado en la mayoría de las naciones del istmo, la integración eléctrica centroamericana se considera la mejor opción para favorecer un desarrollo más eficiente del sector. Esas mejoras derivarían de tres elementos fundamentales: $a$ ) la posibilidad de construir plantas generadoras más grandes con un mejor aprovechamiento de economías de escala y que utilizasen tecnologías más eficientes para la producción de energía (tecnologías no rentables en mercados muy reducidos); ${ }^{4}$ ) la complementariedad hidráulica y de otras fuentes de generación, así como la basada en diferencias en las curvas de carga entre diversos países $;{ }^{5} c$ ) las economías de escala derivadas de una integración de la red de transporte. ${ }^{6}$ Si bien este proceso de integración no se ha detenido desde la firma del acuerdo que lo originara en 1997, su avance es lento y de ahí que su repercusión en las estrategias adoptadas hasta el momento por las ET que operan en la región haya sido escasa.

Con respecto a la situación previa a la reforma, su principal característica fue la fuerte crisis motivada por la falta de inversiones que, unido a la debilidad y subordinación del Estado en la negociación con las ET, favoreció el desarrollo de determinados contratos de compraventa de energía (Power Purchase Agreements, PPAs) en los que se reconocen costes de generación por encima de los estándares internacionales. ${ }^{7}$ Este tipo de contratos ha operado como factor de localización fundamental en el área de generación.

3 Téngase en cuenta que la media del consumo mundial por habitante en ese mismo año fue de 2.085 kwh/habitante, y la de América Latina y el Caribe, 1.452kwh/habitante (Banco Mundial, 2001).

4 Se está haciendo referencia a los ciclos combinados de gas para lo cual se hace necesario la construc ción de un gasoducto desde México. Dicha construcción sería inviable si tuviera que realizarse a distintas centrales instaladas en cada uno de los países del istmo. La situación sería diferentes si, dada la interconexión sin restricciones a las transacciones de energía, se pudieran proyectar plantas con una orientación regional y no local. Un estudio del BID sobre la interconexión centroamericana señala que las centrales térmicas pequeñas que se están instalando en los países con enfoque nacional, han logrado precios de US\$0.055 por kwh mientras que las tecnologías más avanzadas de ciclos combinados que prevalecen en mercados más grandes podrían lograr precios entre US\$0.035 y US\$0.045kwh para unidades de 200 a $250 \mathrm{mw}$. Una planta de $250 \mathrm{mw}$ con estas características, a un precio de US $\$ 0.04$ por kwh, representaría ahorros potenciales de US\$28.0 millones por año, si se la compara con la misma capacidad en varias plantas pequeñas (BCIE/BID/CEPAL, 2001:5).

5 La creación de mercados regionales permite disminuir las necesidades de sobreequipamiento a nivel regional, reduciendo los riesgos derivados de aumentos puntuales en la demanda o falta de suministro por fallos en las centrales o por escasez de lluvias. Además, dado que la curva de demanda no es igual en todos los países, la integración del mercado permite complementar las faltas y los excesos de energía entre ellos (Coral, 2002:5).

6 De acuerdo con el trabajo de Coral (op. cit.) las economías por la ampliación de la red de transporte constan de dos tipos de componentes: una asociada a los costos de los equipos y la otra a la cobertura del servicio: una ocurre cuando se puede aumentar la capacidad de los equipos con un aumento proporcionalmente menor en el coste correspondiente; otra denominada de alcance, ocurre porque la disminución de costos se da al lograr grandes coberturas. 
Tabla 2

Istmo centroamericano: evolución de la demanda máxima de energía Valores absolutos (mw)

\begin{tabular}{llrrrrrr}
\hline Años & Istmo & Costa Rica & El Salvador & Guatemala & Honduras & Nicaragua & Panamá \\
\hline 1985 & 1988.0 & 511.0 & 318.4 & 301.6 & 220.0 & 213.0 & 424.0 \\
1990 & 2614.9 & 682.0 & 412.3 & 452.2 & 351.0 & 253.0 & 464.4 \\
1995 & 3630.5 & 871.9 & 591.7 & 717.2 & 503.5 & 327.0 & 619.2 \\
1996 & 3789.4 & 915.1 & 626.0 & 733.4 & 534.0 & 341.0 & 639.9 \\
1997 & 4116.1 & 964.0 & 666.2 & 820.3 & 605.0 & 354.0 & 706.6 \\
1998 & 4318.3 & 991.1 & 694.3 & 877.3 & 649.5 & 379.7 & 726.4 \\
1999 & 4542.6 & 1060.4 & 718.0 & 962.1 & 661.0 & 386.6 & 754.5 \\
2000 & 4772.4 & 1121.3 & 758.0 & 1017.3 & 702.0 & 396.8 & 777.0 \\
$2001^{\text {a }}$ & 4954.9 & 1136.9 & 734.0 & 1074.6 & 758.5 & 411.6 & 839.3 \\
\hline
\end{tabular}

a Cifras preliminares

Fuente: CEPAL sobre la base de cifras oficiales

En la distribución rural, la precaria situación en la que se encontraban las dos distribuidoras del Instituto Nacional de Electrificación (INDE), Distribuidora de Energía de Occidente (DEOCSA) y Distribuidora de Energía de Oriente (DEORSA), obligaron a introducir elementos adicionales para la atracción de capital extranjero. En primer lugar, se vincula la venta de las distribuidoras a la adjudicación de un Plan de Electrificación Rural (PER), cuya financiación corre a cargo del Estado, a partir de fondos obtenidos con las privatizaciones y otro tipo de recursos externos fundamentalmente provenientes de organismos multilaterales de desarrollo. La empresa que compre las distribuidoras quedará encargada a su vez de la ejecución del PER. Esta fórmula permite a la empresa adjudicataria obtener una rentabilidad por la realización de este plan, a la vez que se asegura el incremento del número de usuarios, sin ser ella quien asuma el coste de dicha operación. En segundo lugar, las dos distribuidoras firman un contrato de suministro de energía con el INDE, el cual les permite obtener energía barata y favorecer así la expansión del consumo en el ámbito rural.

En definitiva, el escaso atractivo que por el reducido tamaño del mercado parece ofrecer el sector eléctrico en Guatemala plantea al gobierno la necesidad de favorecer la atracción de inversión extranjera mediante mecanismos adicionales, como los contratos de generación o la financiación de la expansión de las distribuidoras rurales. Estos resultarán ser factores de localización fundamentales por su influencia en las estrategias y modalidades adoptadas por las ET y en los efectos que ha tenido su entrada en el sector.

$7 \quad$ Una explicación detallada de las principales características de estos contratos se ofrece más adelante en el apartado que analiza los efectos en las tarifas eléctricas. 


\section{Estrategias}

Tanto en el área de distribución como en el de generación, la estrategia fundamental que guió las inversiones de las ET ha sido el acceso a nuevos mercados de servicios recientemente liberalizados y privatizados. Dicha estrategia general, presente en la mayoría de las áreas de servicios, adquiere particularidades derivadas de las especificidades del sector eléctrico y la existencia de factores de localización específicos del caso guatemalteco.

En el sector objeto de estudio, la entrada de la competencia no ha eliminado una fuerte herencia de la estructura industrial anterior, en gran parte gracias al reducido tamaño del mercado guatemalteco. ${ }^{8}$ Ello ha llevado a las principales ET que han invertido en el país a poner en práctica distintas estrategias orientadas al ejercicio del poder de mercado.

En el área de generación, las empresas privadas, en su mayoría ET, han creado la Asociación Nacional de Generadores (ANG), que se ha convertido en el principal mecanismo de defensa de sus intereses. Su objetivo inicial fue la instauración de un frente común para evitar una alteración significativa de los PPAs firmados antes de 1996, que es un ejemplo de cómo ejercer poder de mercado para influir en la fijación de los precios de venta de energía.

En este caso, junto con las empresas distribuidoras, están orientando su estrategia hacia un proceso de reintegración vertical. En el caso del consorcio gestionado por Iberdrola (que ha adquirido la Empresa Eléctrica de Guatemala, S.A., EEGSA) se plantearon dos ejes básicos en la estrategia de entrada:

1) La creación de una comercializadora (integración hacia adelante) para la captación de grandes usuarios. Hacia ellos derivan las compras de energía que le resultan más baratas para poder ofrecer precios competitivos.

2) La inversión en generación propia (integración hacia atrás), que le permitiera ir reduciendo el peso significativo de los contratos existentes y abaratando el coste total de las compras de energía. ${ }^{9}$

En el caso de Unión Fenosa (UF), la situación —en un principio- fue más holgada gracias a la firma del PER y de un acuerdo de suministro de energía con el INDE hasta 2003

8 Algunos trabajos señalan que, en casos de mercados pequeños (potencias instaladas de entre $1000 \mathrm{y}$ $2000 \mathrm{mw}$ ) es aconsejable que la apertura sea parcial, pues es probable que de ella se genere una eficiencia técnica y económica mayor que de la introducción de libre competencia. Además, se cuestiona que, en estos mercados, resulte eficiente la desintegración vertical (Altomonte, 2001:11 y 38 )

9 El proyecto se suspendió tras dos años de trabajo y la compra del terreno porque la casa matriz decidió focalizar todas sus inversiones en México y Brasil al considerarlos mercados más estratégicos (Iberener, 2000). Esto muestra la importancia del carácter transnacional de las empresas en la toma de decisiones. 
a precios menores que los de los contratos de generación anteriormente citados. Sin embargo, a partir de ese momento, las circunstancias serán parecidas a las de Iberdrola, lo cual está motivando que UF se plantee también como parte de su estrategia la inversión en generación propia.

Además de estas tácticas, propias de mercados poco competitivos, en Guatemala es frecuente el desarrollo de inversiones de tipo rentista por parte de la mayoría de las ET, que se relaciona con la existencia de factores de localización, tales como el reducido tamaño del mercado y el contexto previo a la reforma. Este tipo de inversión se caracteriza por establecer un horizonte temporal de recuperación relativamente corto — pues la rentabilidad exigida a la operación es elevada - y por la escasa voluntad de permanencia. Todo ello deriva en la importancia relativa de la filial para la empresa en su conjunto. ${ }^{10}$

Las características técnicas de la inversión realizada se ven afectadas por la adopción de estrategias rentistas. Las empresas generadoras optan por instalar plantas térmicas cuya inversión es menor que la de otras tecnologías y que cuentan con un plazo de recuperación más corto. En la mayoría de los casos se trata de plantas que se pueden desmontar con relativa facilidad en caso de optar por la desinversión. El caso más representativo es el de Enron, que las montó sobre barcazas flotantes.

\section{Modalidades}

La entrada de ET en el sector eléctrico guatemalteco se ha dado a través de dos modalidades claramente diferenciadas entre las áreas de generación y distribución. En la primera, la gran mayoría de ET que han desarrollado actividades lo han hecho mediante la inversión en nuevos activos. Por el contrario, en el área de distribución, la modalidad adoptada ha sido la compra de empresas públicas mediante la fórmula de licitación internacional.

En el caso de las actividades de generación son pocos los informes de los que se dispone acerca de las fuentes de financiación de las inversiones, pero los existentes muestran que es minoritaria la participación de recursos provenientes de la casa matriz y parecen significativos aquellos obtenidos a través de organismos tales como el BID (Banco Interamericano de Desarrollo) o el Banco Mundial (CEPAL, 2001). De hecho, el financiamiento obtenido mediante los mercados financieros internacionales es poco frecuente debido a la alta calificación riesgo-país de Guatemala que encarece mucho el coste de la misma. La modalidad adoptada por Uf en el área de distribución es muy parecida a la anteriormente señalada: aseguró las inversiones en el PER a partir de la participación de recursos provenientes del BID y el BCIE (Banco Centroamericano de Integración Económi-

10 Por ejemplo, en el caso de Unión Fenosa, según datos de la Memoria de 2001, las inversiones en Guatemala representan alrededor de $3.5 \%$ de las realizadas internacionalmente, lo que significa $1.2 \%$ de la actividad total del grupo. En el caso de Iberdrola, según el informe anual del 2000, la inversión en Guatemala representa $7.7 \%$ del total realizado por la compañía en el exterior. 
ca). En décadas pasadas, dichos recursos se destinaban a los proyectos gestionados por las empresas públicas, por lo que más que aumento o mejora en el acceso a fondos externos parece que hubo un cambio en la titularidad de quien los gestiona y, en consecuencia, de la distribución de rentas derivada de dicho cambio.

En algunas de las inversiones de ET en generación, se optó por buscar asociación con capitales locales que, a pesar de que participan minoritariamente, ayudan a reducir riesgos y permiten mejorar el conocimiento del sector y la posición negociadora con el gobierno. En el caso de empresas estadounidenses, aunque ostenten la propiedad de sus inversiones, suelen ceder la gestión a empresarios locales, modalidad que busca objetivos muy similares a los anteriormente citados. Por el contrario, en el caso de las empresas españolas, la gestión la realizan directivos de esa misma nacionalidad, lo cual facilita la implantación de su know how, pero supone menores ventajas en cuanto al conocimiento del sector y la capacidad negociadora con el gobierno o con empresarios locales.

Tabla 3

ET en el sector eléctrico: factores de localización, estrategias y modalidades

\begin{tabular}{|c|c|c|}
\hline Factores de localización & Estrategias & Modalidades \\
\hline \multicolumn{3}{|l|}{ Generales } \\
\hline $\begin{array}{l}\text { - Proceso de reformas: } \\
\text { liberalización y privatización }\end{array}$ & $\begin{array}{l}\text { - Estrategia principal: acceso a } \\
\text { mercados de servicios } \\
\text { recientemente liberalizados }\end{array}$ & $\begin{array}{l}\text { - Inversiones en generación: } \\
\text { construcción de nuevos activos. } \\
\text { Financiación compartida con } \\
\text { organismos multilaterales o con } \\
\text { empresarios locales }\end{array}$ \\
\hline $\begin{array}{l}\text { - Contexto político-institucional: } \\
\text { características del estado regulador }\end{array}$ & $\begin{array}{l}\text { - Estrategias dérivadas de la } \\
\text { especificidad del sector: estra- } \\
\text { tegias de poder de mercado: } \\
\text { i) Asociación entre empresas } \\
\text { ii) Reintegración vertical }\end{array}$ & $\begin{array}{l}\text { - Inversiones en distribución: } \\
\text { adquisición de activos ya existentes } \\
\text { (procesos de privatización). Ma- } \\
\text { yor participación de recursos pro- } \\
\text { venientes de la casa matriz }\end{array}$ \\
\hline $\begin{array}{l}\text { - Crecimiento de la demanda y } \\
\text { déficit energético }\end{array}$ & $\begin{array}{l}\text { - Estrategias derivadas de la } \\
\text { especificidad del país: estrategias } \\
\text { rentistas: estrategias de precios, } \\
\text { de tecnologías de generación, } \\
\text { nuevas inversiones }\end{array}$ & \\
\hline - Estrechez del mercado & $\begin{array}{l}\text { - Estrategias de generación vs } \\
\text { distribución: precio de la energía } \\
\text { vs extensión de la cobertura del } \\
\text { suministro }\end{array}$ & \\
\hline \multicolumn{3}{|l|}{$\begin{array}{l}\text { - Ausencia de recursos energéticos } \\
\text { primarios }\end{array}$} \\
\hline $\begin{array}{l}\underline{\text { Específicos }} \\
\text { - Contratos de generación } \\
\text { - PER }\end{array}$ & & \\
\hline
\end{tabular}

Fuente: elaboración propia 
En las inversiones realizadas por las compañías distribuidoras sí hay un mayor aporte de recursos propios, en parte por un menor acceso a la financiación de la banca multilateral, pero también por ser una estrategia habitual de estas empresas españolas.

La procedencia de los fondos invertidos marca una diferencia significativa entre unas compañías y otras. Las inversiones de estadounidenses en Guatemala, realizadas mayoritariamente con recursos ajenos, están aseguradas por entidades privadas y públicas, estadounidenses e internacionales, que reducen los riesgos inherentes a la operación y favorecen las posibilidades de desinversión. En el caso de las españolas, al comprometer bienes propios, las pérdidas en caso de desinversión son mayores.

Tras observar los principales elementos que han caracterizado la expansión de ET en el sector eléctrico guatemalteco (véase tabla en página anterior), ahora, en el siguiente apartado, haremos el análisis de los efectos.

\section{Efectos}

Una vez descritas las características de la expansión llevada a cabo por ET, a continuación se aborda el análisis de los efectos que dicha expansión ha producido en las siguientes variables: tarifas, garantía y calidad del suministro y electrificación rural.

\section{Tarifas}

Uno de los objetivos fundamentales de los programas de liberalización y privatización del sector eléctrico es la reducción de los precios de la energía. En teoría, esto sería posible gracias a la sustitución del monopolio por un mercado libre regido por el ejercicio de la competencia. Veamos qué ha ocurrido en el caso guatemalteco.

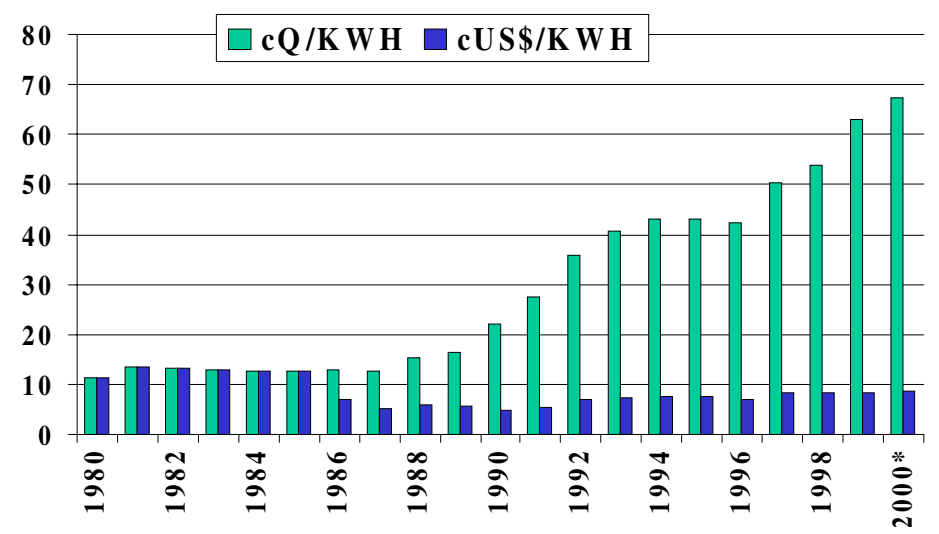

Gráfica 1. Precio medio del consumo de energía eléctrica (EEGSA).

* Datos de agosto de 2000.

Fuentes: BANGUAT; CNEE; tipo de cambio por COPADES. 
Un análisis de los datos que aparecen reflejados en la Gráfica 1 permite comprobar el fuerte aumento experimentado por las tarifas eléctricas guatemaltecas desde que se inicia la participación de la generación privada.

El valor final de las tarifas está formado por tres componentes: costes de generación, transporte y distribución. Los primeros representan entre 60 y $70 \%$ del precio final de la energía, los segundos entre 5 y $8 \%$ y los terceros entre 20 y $30 \%$. A esto hay que añadir los impuestos y otros gastos que no suelen representar más de $3 \%$ de la tarifa. Por tanto, el valor final de la misma depende, en gran parte, de los precios de generación, los cuales son, además, los únicos que se establecen por el libre funcionamiento de las fuerzas del mercado. ${ }^{11}$ Por ello, el análisis se va a centrar en lo ocurrido en esta actividad.

\section{Mecanismos para la compra de energía}

El mercado de la electricidad en Guatemala ofrece tres mecanismos para la compraventa de energía: el mercado a término, el de oportunidad y el de desvíos de potencia. En el primer caso, se trata de un acuerdo bilateral donde los términos de las transacciones (cantidad y precio) quedan definidos. En el segundo, las ofertas y demandas acuden conjuntamente a un mercado en el que un administrador se encarga de casarlas estableciendo un único precio final para todas ellas. El precio de la energía en este mercado corresponde al coste marginal de corto plazo (СMCP) de la última unidad generadora despachada. El tercer mecanismo permite gestionar las faltas o excesos de potencia a un precio fijo establecido por el administrador.

El mercado a término representa un porcentaje creciente de las transacciones en los últimos años: $83.5 \%$ en 2000 y $87 \%$ en 2001(Амм, 2000 y 2001). Dentro de ese mercado a término, los PPAs (contratos de compraventa de energía suscritos con anterioridad a la entrada en vigor de la Ley General de Electricidad [LGE] aprobada en 1996) representan un porcentaje mayoritario. En concreto, la producción realizada bajo los PPAs de ET representó $30.1 \%$ de las ventas de energía en 2000 y 38.1 en $2002 .{ }^{12}$

Partiendo de estos datos parece razonable plantear que el precio final de la energía eléctrica en Guatemala ha estado condicionado de forma creciente por el precio derivado de los contratos PPAs, en su mayoría firmados por ET. Por ello, a continuación se analizan sus principales características.

11 Los peajes de transmisión y el VAD son calculados por la CNEE en función de los costes medios de capital y de operación de una red de transporte y distribución con las características de la guatemalteca.

12 Estos porcentajes están subestimados debido a la inclusión dentro de las ventas del INDE de las realizadas por las geotérmicas y algunas hidráulicas. Como ya se ha mencionado, en 2003, el INDE asumió los PPAs que firmaron las distribuidoras DEOCSA y DEORSA. El INDE compra la energía a un precio superior al establecido en el contrato de suministro con UF. El resto de la energía también la vende en el mercado a un precio por debajo del de su compra para tratar de aminorar la subida de los precios. 
LOS PPAS

En el periodo 1992-1997 se firmaron un total de 20 contratos, once correspondientes a la EEGSA y nueve al INDE. Los de EEGSA representan un compromiso de compra de capacidad por 645Mw, de los cuales $564(87 \%)$ corresponde a ET.

Tabla 4

Guatemala: evolución reciente de las ventas en el mercado mayorista de electricidad (gwh)

\begin{tabular}{|c|c|c|c|c|}
\hline \multirow[t]{2}{*}{ Empresas } & \multicolumn{2}{|c|}{2000} & \multicolumn{2}{|c|}{2001} \\
\hline & Valor absoluto & Porcentaje & Valor absoluto & Porcentaje \\
\hline Total oferta & 6069.5 & & 5825.2 & \\
\hline Contratos & 5068.7 & 83.5 & 5061.3 & 86.9 \\
\hline INDE & 2411.6 & 39.7 & 2281.2 & 39.2 \\
\hline Enron (PQPC y Poliwatt) & 675.9 & 11.1 & 908.9 & 15.6 \\
\hline Cogeneradores & 651.4 & 10.7 & 567.1 & 9.7 \\
\hline SIDEGUA & 130.3 & 2.1 & 90.5 & 1.6 \\
\hline GGG & 500.5 & 8.2 & 446.2 & 7.7 \\
\hline Tampa & 99.6 & 1.6 & 20.3 & 0.3 \\
\hline San José & 556.7 & 9.2 & 845.5 & 14.5 \\
\hline Otros & 42.8 & 0.7 & -95.5 & -1.6 \\
\hline Mercado de ocasión & 100.8 & 1.7 & 764 & 13.1 \\
\hline INDE & 470.6 & 7.8 & 166.4 & 2.9 \\
\hline Comegsa & 156.7 & 2.6 & 8.9 & 0.2 \\
\hline Poliwatt & 122.9 & 2.0 & 178.4 & 3.1 \\
\hline Genor & 14.4 & 0.2 & 10.2 & 0.2 \\
\hline GGG & 19.2 & 0.3 & 2.1 & 0.0 \\
\hline CECSA & 39.4 & 0.6 & 4.6 & 0.1 \\
\hline CEISA & 38.7 & 0.6 & 27.9 & 0.5 \\
\hline CONEC & 13.7 & 0.2 & 0 & 0.0 \\
\hline MEL & 39.2 & 0.6 & 13.2 & 0.2 \\
\hline Otros & 86.0 & 1.4 & 352.4 & 6.0 \\
\hline
\end{tabular}

El sombreado distingue a las ET.

Fuente: administrador del mercado mayorista.

En el caso de los contratos del INDE, que representan compromisos por $67 \mathrm{mw}$ de potencia, sólo está clara la participación de una ET en el caso de la geotérmica de Orzunil (24Mw). La importancia de estos contratos reside no sólo en que tienen un porcentaje mayoritario y niveles cercanos a $40 \%$ de la energía generada, sino que además fueron el punto de partida para la firma de otros similares con generadores locales, fundamentalmente con los ingenios azucareros.

Algunas de las características que se van a exponer a continuación se han modificado por la renegociación de los contratos que tuvo lugar en 2001. Sin embargo, dado que estuvieron vigentes hasta esa fecha y que los cambios no han afectado sustancialmente los 
términos de los contratos, se presentarán en primer lugar los más destacados, y más tarde se aclararán las citadas modificaciones que se llevaron a cabo: ${ }^{13}$

a) Todos los contratos están indexados a los precios internacionales de los combustibles. Salvo en el caso de Tampa y, parcialmente en el de San José, la indexación se hace sobre el total de costos variables que incluye además del cargo por combustibles, los costes de operación y mantenimiento. No obstante, la evolución de dichos costes es independiente de la de los precios internacionales de los combustibles.

b) En todos los contratos se reconoce de forma separada los cargos por potencia (costes fijos) y por energía (costes variables).

Cargos por potencia o capacidad contratada. En los cargos por potencia se reconoce un incremento anual de entre 2 y $3 \%$ a pesar de que el nivel de partida representaba valores muy por encima de los estándares internacionales. Según un informe de consultoría externa contratado por la Comisión Nacional de la Energía Eléctrica (CNEE) (SYNEX, 1997:2), el cargo por potencia estándar para ese tipo de centrales se situaba alrededor de 6 US\$kw/ mes, mientras que en el contrato se reconocieron los niveles base de 17 y 13.05 US\$ para Enron y Tampa, respectivamente (ibid). ${ }^{14}$

Cargos por energía o cargos por operación y mantenimiento (СОм). La evolución de éstos depende de los precios internacionales de los combustibles, el tipo de cambio (los contratos están referidos en dólares aunque son abonados en quetzales) y la inflación de Eu. Además de la problemática derivada de la consideración de los costes de forma unitaria, las repercusiones en el precio de las tarifas se derivan de un sobredimensionamiento de los mismos. En la Tabla 4 se aprecian las diferencias entre los costes no asociados a combustible reflejados en los contratos y los estándares para este tipo de centrales.

Esto refleja que el impacto en las tarifas no se debe sólo a la subida del precio del petróleo o a las fluctuaciones del tipo de cambio, sino a un sobredimensionamiento de los costes (tanto los de potencia como los de energía) reconocidos en contrato. Además, la consideración de los com de forma unitaria permite beneficios adicionales a la subida de

13 La información sobre el contenido de los PPAs se ha extraído fundamentalmente de dos documentos de la CEPAL (2001a y 2001c) a los que se puede acudir para ampliar la información sobre estos mismos contratos y sobre los restantes.

14 Para establecer los cargos por capacidad es necesario determinar la que tiene cada planta. En la mayoría de los contratos se obliga al pago de la capacidad estipulada en los mismos, aún cuando en la realidad ésta se reduzca. De acuerdo con las opiniones de diversos analistas del sector (CEPAL, 2001c:35 y entrevistas agosto noviembre de 2001), los procedimiento utilizados para realizar este cálculo en las distintas plantas fueron muy favorables a los productores. De hecho, es frecuente que la capacidad realmente ofertada fuera inferior a la establecida en el contrato a pesar de lo cual el pago que ha tenido que realizar la distribuidora no se viera afectado. En el argot del sector, a esta situación se le ha denominando como compras de "1 kilo por 800 gramos". 
Tabla 5

Cargos por operación y mantenimiento

no asociados a combustible (US\$/kwh)

\begin{tabular}{llc}
\hline Empresas & Contratos & Estándares $^{a}$ \\
\hline Enron & 0.0151 & 0.0059 \\
GGG & $0.0050^{\mathrm{b}}$ & 0.0052 \\
Tampa & 0.0120 & 0.0099 \\
Orzunil & 0.0114 & 0.0055 \\
\hline
\end{tabular}

a Datos basados en información de la Comisión Federal de Electricidad de México

b Estimación a partir de los valores fijados en los contratos y las eficiencias típicas en centrales similares

Fuente: CEPAL (2001c: 44)

los precios del petróleo, ya que eleva la parte de los сом no afectada por los precios de los combustibles internacionales. El descenso de estos precios reduciría los beneficios de las generadoras pero no crearía problemas serios en la medida en que los niveles de partida se hallan sobredimensionado.

Desde que el consorcio gestionado por Iberdrola compra la EEGSA, comienza a plantearse la necesidad de renegociar los contratos. Finalmente, tras un complicado proceso de negociación, las modificaciones más importantes a las que se llega son las siguientes:

- Entrega durante la hora pico (de 18:00 a 20:00 hrs) como obligación del generador, de toda su potencia contratada, ya sea mediante unidades propias o contratos con terceros (se suprimen los kilos de 800 gramos).

- Eliminación en el escalonamiento del precio de la potencia, estableciéndose su valor a partir del precio de 1998.

Si se tiene en cuenta que al sobredimensionamiento inicial se le añaden los escalonamientos incorporados hasta 1998, se puede entender que los cargos por potencia siguen manteniendo valores por encima del estándar. Los valores de estos cargos para Enron y Tampa se situaron en 1998 en 19.73 y 14.26 (ibid).

A cambio de estas concesiones, los contratos se prorrogan hasta el periodo 2015-2020, de los cuales la mayoría de ellos vence alrededor del 2007. Por tanto, teniendo en cuenta el limitado alcance de las modificaciones realizadas, es previsible que se mantenga la influencia descrita de dichos contratos en los precios de las tarifas. En la medida en que los incrementos en la demanda de energía sean significativos y permitan la entrada en funcionamiento de otras plantas con mecanismos de compraventa más competitivos, el impacto en las tarifas de los contratos existentes será cada vez más reducido. 


\section{Estrategias de las distribuidoras y respuesta del Estado ante los PPAS}

\section{Las distribuidoras}

Frente a la situación descrita anteriormente, las distintas distribuidoras han desarrollado estrategias que les permitan minimizar el impacto negativo de los elevados precios de la energía y expandirse en el mercado eléctrico guatemalteco.

Así, uf condicionó la compra de las distribuidoras del INDE a la suscripción de contratos de suministro de energía que, al menos por un tiempo, le garantizara las compras a un precio menor del que tendría que asumir en ausencia de dicho contrato. Esta situación le ha permitido vender energía a un precio promedio en 2000 de 0.05 US\$/kwh frente al promedio de $0.125 \mathrm{US} \$ / \mathrm{kwh}$ al que vende la EEGSA. Pero esto también tiene un impacto en la cuenta de resultados del INDE, pues está vendiendo energía por debajo del precio al que lo compra.

En el caso del consorcio gestionado por Iberdrola (EEGSA) la situación era más delicada pues su dependencia de PPAs era mucho mayor. Según la ley, los usuarios finales de energía se dividen en regulados (UR) y no regulados (UNR). ${ }^{15}$ EEGSA tiene suscritos PPAs por una potencia de $645 \mathrm{mw}$, cantidad que distribuía entre todos los usuarios (regulados y no regulados) de la empresa. Con la aprobación de la LGE se abre la competencia en el sector de UNR, lo cual significa que éstos ya no están obligados a comprar a la EEGSA y pueden hacerlo a cualquiera de las comercializadoras. Dado el elevado coste de la energía suministrada por esta empresa como consecuencia de los compromisos de compra adquiridos, el riesgo de perder a UNR era muy elevado.

Ante esta situación, la EEGSA decide montar su propia comercializadora para abastecer a los UNR y repercutir sobre los regulados la mayor cantidad posible de contratos PPAs. Es decir, los mayores precios de la energía ya no se reparten proporcionalmente entre todos los usuarios, sino que los soportan en su mayoría los regulados. ${ }^{16}$ Cualquier incremento de la demanda en este sector es suministrado mediante los PPAs. Esto le permite a la comercializadora ofrecer en el sector de los no regulados (donde hay mayor competencia) menores precios, mientras que los regulados (sometidos a tarifa) asumen todo el sobrecosto de los PPAs. Para hacerse una idea de las diferencias entre la distribuidora y la comercializadora, téngase en cuenta que en 2001, la primera adquirió en el mercado de oportunidad $1.89 \%$ de la energía que suministró, y la comercializadora obtuvo en ese mismo mercado $31.34 \%$ (Амм, 2001). En cada caso, el resto fue adquirido mediante contratos a término.

15 Los primeros hacen referencia a pequeños consumidores, normalmente residenciales o comerciales, mientras que los segundos incluyen el sector industrial y otro tipo de empresas como hoteles, centros comerciales, entre otros.

16 Téngase en cuenta que aunque sean regulados, las tarifas se elaboran mediante el mecanismo denominado pass through, lo cual permite repercutir íntegramente el coste de la energía. 


\section{El Estado}

Frente al aumento sostenido en los precios de las tarifas al sector regulado, el Estado se plantea la adopción de diferentes mecanismos que permitan contrarrestar dicha subida. Por una parte, el INDE firma el citado contrato de suministro con las distribuidoras rurales. Además, en 1999, el Estado autoriza al INDE a vender por debajo del valor de mercado la energía acumulada por las favorables condiciones hidrológicas. Finalizada la época de lluvias, el problema persistió, por lo cual, a principios de 2000, el gobierno aprobó un subsidio a los usuarios cuyo consumo fuera inferior a $650 \mathrm{kw} / \mathrm{h}$ al mes (aproximadamente $90 \%$ de ellos). Esto implica que se está subvencionando no sólo al usuario residencial de bajos ingresos, sino también al de altos ingresos e incluso a pequeños comercios. En el año 2001 se aprueba un nuevo subsidio, esta vez para consumos inferiores a los 300kw/h al mes (nivel que se sigue considerando elevado), cuya tarifa pasa a denominarse social. Para el pago de este subsidio se están utilizando recursos propios del INDE y del Estado provenientes de las privatizaciones, lo cual supone una merma considerable de estos ingresos.

En definitiva, el análisis realizado en este apartado muestra que el impacto en las tarifas como consecuencia de la entrada de ET en el sector eléctrico guatemalteco es negativo. Esta valoración se justifica por el significativo aumento experimentado en el precio de la energía, que se relaciona directamente con las condiciones de los contratos firmados por las ET. Además, este incremento obligó al Estado a intervenir subsidiando las tarifas a una parte mayoritaria de la población. Esta situación implica no sólo que no se está cumpliendo con el objetivo de reducción de las tarifas, sino que además se está incurriendo en una pérdida de recursos del Estado. Esto contrarresta, al menos en parte, los posibles efectos benéficos en términos fiscales que se presupone se derivarán de las privatizaciones.

\section{Garantía y calidad del suministro}

El aumento de la capacidad instalada y la mejora de la eficiencia han constituido también objetivos fundamentales del proceso de reformas. La entrada de recursos económicos por medio de las privatizaciones y la llegada de IED supondrían un aumento de las inversiones en generación así como la transferencia de tecnología y de las ventajas competitivas de las ET. Estos serían los instrumentos que permitirían el cumplimiento de los objetivos apuntados. Veamos nuevamente qué es lo que ha ocurrido en el caso guatemalteco.

\section{Garantía del suministro: generación}

En los últimos años, el indicador de fiabilidad del sistema eléctrico ha mejorado gracias al menor crecimiento del consumo y a la entrada en funcionamiento de nuevas plantas de generación. Aunque ese indicador no ha alcanzado los niveles de 1985, se encuentra en una situación aceptable e incluso más ajustada a las necesidades del sistema, un indicador 
excesivamente alto no es positivo pues refleja que existe demasiada capacidad ociosa que encarece el precio de la energía.

Tabla 6

Guatemala: Indicador de fiabilidad del sistema eléctrico

\begin{tabular}{|c|c|c|c|c|c|c|}
\hline \multirow[t]{2}{*}{$A \tilde{n} o s$} & \multicolumn{2}{|c|}{ Capacidad instalada $M W(1)$} & \multicolumn{2}{|c|}{ Demanda máxima $M W(2)$} & \multicolumn{2}{|c|}{ Fiabilidad (1/2) } \\
\hline & Guatemala & Centroamérica & Guatemala & Centroamérica & Guatemala & Centroamérica \\
\hline 1980 & 366.5 & - & 273 & - & 1342 & - \\
\hline 1985 & 783.4 & 3931.4 & 301.6 & 1988.0 & 2597 & 1978 \\
\hline 1990 & 810.9 & 4129.3 & 452.2 & 2614.9 & 1793 & 1579 \\
\hline 1995 & 1082.3 & 5218.4 & 717.2 & 3630.5 & 1509 & 1437 \\
\hline 1996 & 1145.5 & 5434.8 & 733.4 & 3789.4 & 1562 & 1434 \\
\hline 1997 & 1205.7 & 5625.5 & 820.3 & 4116.1 & 1470 & 1367 \\
\hline 1998 & 1313.2 & 5966.5 & 877.3 & 4318.3 & 1497 & 1382 \\
\hline 1999 & 1463.0 & 6573.3 & 962.1 & 4542.6 & 1521 & 1447 \\
\hline 2000 & 1668.3 & 7256.5 & 1017.3 & 4772.4 & 1640 & 1521 \\
\hline 2001 & 1672.1 & 7399.2 & 1074.6 & 4954.9 & 1556 & 1493 \\
\hline
\end{tabular}

Fuente: elaboración propia a partir de datos del INDE y el AMM.

Partiendo de este aumento de la capacidad instalada y de su importancia para garantizar el suministro eléctrico, es necesario profundizar en las características e implicaciones de estas nuevas inversiones.

a) Mix eléctrico. El aumento de la capacidad instalada se ha llevado a cabo mediante una diversificación del mix eléctrico, es decir, de las tecnologías utilizadas para la producción de electricidad. Hasta principios de los noventa, un porcentaje mayoritario de la energía producida en Guatemala provenía de plantas hidroeléctricas, situación que cambió drásticamente en los últimos años (véase Tabla 6).

Tabla 7

Guatemala: estructura de la capacidad instalada (mw)

\begin{tabular}{lrrrrr}
\hline & Total & Hidro & Geo & Térmicas & Cogeneración \\
\hline 1985 & 783.4 & 488.1 & - & 295.3 & - \\
1990 & 810.9 & 488.1 & - & 322.8 & - \\
1995 & 1082.3 & 502.1 & - & 525.2 & 55 \\
1996 & 1145.5 & 502.1 & - & 563.4 & 80 \\
1997 & 1205.7 & 496.5 & - & 569.2 & 140 \\
1998 & 1313.2 & 512.6 & 5.0 & 655.2 & 140.4 \\
1999 & 1463.0 & 510.8 & 29.0 & 782.8 & 140.4 \\
2000 & 1668.3 & 530.9 & 29.0 & 944.7 & 163.7 \\
2001 & 1672.1 & 524.9 & 33.0 & 914.2 & 200 \\
\hline
\end{tabular}

Fuente: CEPAL, sobre la base de cifras oficiales. 
A pesar de que este hecho se puede considerar positivo desde la perspectiva de la garantía del suministro — reduce la inestabilidad y el riesgo de escasez cuando el régimen de lluvias es bajo-, no ocurre lo mismo si se analiza desde otras variables como el precio de la energía, el impacto medioambiental e incluso la cuestión fiscal.

b) Medio ambiente. Desde el punto de vista medioambiental, el impacto ha sido negativo debido a que las tecnologías térmicas utilizadas son altamente contaminantes. En Guatemala aumentó la capacidad instalada a partir de la generación mediante las tecnologías con mayor impacto medioambiental, las térmicas que utilizan como combustible derivados del petróleo (IDAE, 2001). La falta de legislación laboral y de controles permite el desarrollo de centrales térmicas que utilizan tecnologías obsoletas con elevados niveles de emisiones muy por encima de los estándares permitidos en otros países. El propio Ministerio de Medio Ambiente reconoció que la legislación en Guatemala es, en este sentido, muy laxa, cuestión que se atribuye a la necesidad de evitar trabas a los inversionistas. A priori, la llegada de ET facilita el acceso a recursos para la inversión en aumento de la capacidad instalada. Sin embargo, las características del país y la falta de competencia dentro del sector desincentivan a las ET a realizar inversiones en otro tipo de tecnología de generación más moderna.

c) Dependencia energética y fiscalidad. La mayoría de las centrales térmicas utilizan como combustible derivados del petróleo. Además de las consecuencias medioambientales, esto supone un aumento considerable de la dependencia energética que se refleja en el crecimiento sostenido de las importaciones de hidrocarburos. Hasta el momento, Guatemala no ha puesto en marcha ninguna política específica para abordar esta cuestión. Por el contrario, en el sector eléctrico ha aplicado una serie de medidas que favorecen la utilización de combustibles derivados del petróleo. De acuerdo con el decreto 38-92 y el Acuerdo Gubernativo 359-96, las empresas generadoras quedan exentas del pago de impuestos por las importaciones de combustibles. Teniendo en cuenta que la cuantía de este impuesto

Tabla 8

Guatemala: consumo de combustibles de plantas generadoras (miles de galones) y ahorro de impuestos (miles de quetzales)

\begin{tabular}{|c|c|c|c|c|c|c|c|c|}
\hline & 1993 & 1994 & 1995 & 1996 & 1997 & 1998 & 1999 & 2000 \\
\hline \multicolumn{9}{|l|}{ Búnker } \\
\hline - Consumo & 36565 & 50695 & 49439 & $62 \quad 274$ & 115044 & 115895 & 96667 & 101879 \\
\hline - Ahorro & 18282 & 25347 & 24719 & 31137 & 57522 & 57947 & 48333 & 50939 \\
\hline \multicolumn{9}{|l|}{ Diesel } \\
\hline - Consumo & - & - & 5913 & 14296 & 22036 & 24678 & 12216 & 7455 \\
\hline - Ahorro & - & - & 4435 & 10722 & 16527 & 18509 & 9162 & 5591 \\
\hline
\end{tabular}

Fuente: elaboración propia a partir de datos sobre importaciones y consumos de combustibles de Ministerio de Energía y Minas. 
era de 0.5 quetzales para el caso del búnker C y 0.75 para el caso del diesel, principales combustibles usados por los generadores en Guatemala, en el periodo 1993-2000 los generadores ahorraron más de 379 millones de quetzales (unos 50 millones de dólares) gracias a dicha exención.

d) Relación con la infraestructura de transporte. La forma en que se llevó a cabo el aumento en la capacidad instalada creó problemas también con las redes de transporte. Como se ha visto, casi la totalidad de las inversiones realizadas por ET en cuanto a generación en Guatemala se ha aplicado a centrales térmicas que utilizan como combustible derivados del petróleo. Todas ellas lo importan y es suministrado, en su mayoría, por compañías estadounidenses y transportado en barcos, lo cual llevó a las empresas a localizar las plantas en la cercanía de un puerto para evitar el transporte del combustible hasta éstas.

Existe una elevada concentración de centrales en las inmediaciones del puerto del Pacífico (30\% de la capacidad instalada total, que aumentará con la finalización del proyecto de Duke) que supone una sobrecarga para las líneas de transporte y las subestaciones que conectan ese punto con la capital del país, principal centro de consumo. Esto implica una serie de restricciones al transporte que impiden en determinadas horas del día el uso de la energía proveniente de estas plantas y obligan a utilizar la proveniente de otras, ubicadas en lugares más descongestionados, situación que provocó una pérdida de eficiencia del sistema que se manifestó en el incremento de las tarifas ("sobrecostos por generación forzada"). Éstos hacen referencia a la necesidad de utilizar la energía proveniente de plantas menos eficientes debido a restricciones en las redes de transporte. Por otra parte, como las centrales de Tampa, San José o Enron tienen contratos take or pay, el sistema está pagando una potencia de la que realmente no puede hacer uso. De acuerdo con la información suministrada por el INDE, estos sobrecostos supusieron sólo en los primeros siete meses de 2001 unos 8 millones de dólares. Con respecto a la ley, son los generadores quienes deben hacerse cargo de este coste a manera de incentivo para invertir en transporte o para planificar su ubicación acorde con los requerimientos de eficiencia del sistema y no sólo a los de su central. Sin embargo, en la práctica, la existencia de los PPAs y la falta de competencia en el sector impide que se dé de esta forma, por lo que el Estado terminó por asumir estos costes para evitar mayores incrementos en el precio de la energía.

\section{Calidad del suministro: distribución}

En el ámbito de la distribución, las características de la actividad determinan que siga siendo un monopolio natural, aunque privado. Por tanto, no existe un entorno competitivo que incentive la transferencia de las ventajas que se presuponen a las empresas que se han hecho cargo de la distribución. En estas circunstancias, dicha transferencia va a depender de las estrategias adoptadas por las ET en función del marco regulatorio existente, del 
papel del organismo regulador, y del volumen y aplicación de las sanciones relacionadas con la calidad de la distribución.

De acuerdo con las Normas Técnicas del Servicio de Distribución (NTSD) de la CNEE se establecen cuatro dimensiones para medir la calidad del servicio de distribución eléctrica: calidad del producto, del servicio técnico, del servicio comercial y la atención al cliente. En los dos últimos casos, dada la inexistencia de niveles de referencia y la falta de indicadores en la etapa previa a la reforma, la valoración es prácticamente imposible. En los dos primeros, sí se han establecido parámetros que permiten valorar la evolución reciente de la calidad del suministro. Éstos fueron determinados mediante negociación con las propias compañías teniendo en cuenta la situación en la que se encontraba el sistema de distribución guatemalteco en cada una de las áreas geográficas. ${ }^{17}$

Con respecto a los indicadores de calidad del producto, el último informe disponible de labores de la CNEE no incluía aún datos sobre los mismos por lo que el análisis se centra exclusivamente en los indicadores de calidad del servicio técnico. En la Tabla 8 se muestra la evolución por semestres de los dos principales indicadores utilizados por la CNEE para valorar y sancionar la calidad del servicio técnico realizada por las empresas distribuidoras.

Tabla 9

Guatemala: indicadores de calidad del suministro (FMIK у TTIK $)$

\begin{tabular}{lcclcrrrr}
\hline & \multicolumn{2}{c}{ NMR $^{\mathrm{b}}$} & \multicolumn{2}{c}{$2-2000$} & \multicolumn{2}{c}{$1-2001$} & \multicolumn{2}{c}{$2-2001^{\mathrm{c}}$} \\
\hline & $F M I K$ & TTIK & FMIK & TTIK & FMIK & TTIK & \multicolumn{1}{c}{ FMIK } & \multicolumn{1}{c}{ TTIK } \\
EEGSA & 3 & 10 & 7.49 & 5.99 & 5.20 & 4.36 & 6.80 & 4.33 \\
DEOCSA & 4 & 15 & - & - & 8.07 & 26.06 & 14.97 & 34.76 \\
DEORSA & 4 & 15 & - & - & 13.94 & 30.38 & 28.48 & 57.34 \\
\hline
\end{tabular}

a El TTIK mide el tiempo total de interrupción por kwh instalado y el FMIK mide la frecuencia media de interrupción por kwh.

b Niveles máximos de referencia en la etapa de transición. Hace referencia a los niveles máximos que pueden alcanzar las distintas empresas.

c En el segundo semestre de 2001 EEGSA entra ya en la etapa de régimen en que los niveles de referencia son 2.5 y 8 para el FMIK y el TTIK respectivamente.

Fuente: elaborado a partir de información de la CNEE.

Como se aprecia en la tabla, ninguna de las distribuidoras cumplen los niveles requeridos. La desviación es especialmente acusada en las distribuidoras del interior, en las cuales además se aprecia un considerable empeoramiento de un semestre a otro. El incumplimiento de los niveles requeridos está relacionado con las características del sistema san-

17 En el artículo 4 de la normativa (NTSD Resolución de la CNEE núm. O9 99) se establece la existencia de cuatro etapas diferenciadas (denominadas preliminar, prueba, transición y régimen) para posibilitar una adecuación gradual de las empresas a las exigencias establecidas. Sólo a partir de la última etapa (en la que EEGSA entró a mediados de 2001 y DEOCSA y DEORSA en enero de 2002), los incumplimientos serán sancionados con multas. 
cionador. En ocasiones, a las empresas les resulta más barato pagar las multas que mejorar los indicadores.

Aunque las empresas distribuidoras han incorporado diversos avances en los métodos de gestión y control de las redes, una mejora significativa en la calidad del servicio requiere inversiones en las propias redes físicas. En el caso de las distribuidoras rurales, los peores resultados en términos de calidad se relacionan precisamente con la estrategia de entrada en Guatemala basada en la expansión del número de usuarios a través del PER. Esto ha ocasionado que la actividad de la compañía se centre en la expansión de la red y no en su mejora. La alta dispersión de los usuarios y el bajo consumo de muchos de ellos condiciona que las dos distribuidoras rurales centren su estrategia en el suministro a grandes clientes pues, en el caso de las pequeñas aldeas, les resulta más barato pagar la multa que mejorar el servicio.

\section{Electrificación rural}

La privatización de las empresas distribuidoras del INDE incluía el compromiso — por parte de la empresa compradora- de llevar a cabo un plan de electrificación rural en el país. Para su desarrollo se establecería un fideicomiso con valor de 333.6 millones de US\$ que provendrían de los ingresos recibidos por privatizaciones, fondos del Estado guatemalteco y préstamos concedidos por organismos internacionales. En la Tabla 10 se muestra la estructura de los fondos según su procedencia.

El contenido y la forma con que se ha planteado el desarrollo del PER trata de cubrir dos objetivos: $a$ ) avanzar en el proceso de electrificación del país, uno de los más bajos de la región; $b$ ) garantizar a la empresa compradora de las distribuidoras rurales la expansión de su área de negocio sin riesgo alguno para la misma. De hecho, durante el tiempo que

Tabla 10

Estructura del fideicomiso

\begin{tabular}{lrl}
\hline & $\begin{array}{c}\text { Monto } \\
\text { (millones de US\$) }\end{array}$ & \multicolumn{1}{c}{ Procedencia } \\
\hline Realizados & 101.0 & - Venta de $80 \%$ de las acciones de las distribuidoras del INDE (4/05/99) \\
& 51.0 & - Bonos del Tesoro $(05 / 01 / 2000)$ \\
Proyectados & 6.3 & - Venta 5\% acciones restantes a UF (3/11/2000) \\
& 10.9 & - Intereses del fideicomiso devengados \\
& 40.0 & - Préstamo del BCIE (en trámite) \\
& 90.0 & - Préstamo del BID (concedido) \\
& 19.0 & - Intereses del fideicomiso (años restantes) \\
2.8 & - Venta acciones a empleados (5.51\% capital) \\
& 12.0 & - Venta dispersa remanente de acciones
\end{tabular}

a En mayo de 2002 el BID aprueba la concesión del préstamo al gobierno guatemalteco para la continuación de las obras del PER.

Fuente: INDE. 
duran las obras del PER, éstas se constituyen en la principal fuente de ingresos de las empresas distribuidoras privatizadas.

Este intento de integrar las necesidades del país con las estrategias de expansión de UF ha sido posible gracias a la participación del Estado como principal fuente de aportación de capital. De los 333.6 millones utilizados en el PER, 173.1 (52\%) provienen directamente de fondos propiedad del Estado y $39 \%$ de préstamos concedidos a éste por organismos multilaterales. Es importante señalarlo porque una de las razones que justificaron la privatización de las distribuidoras del INDE era la falta de recursos para acometer un PER. De los 333.6 millones que se van a invertir en el desarrollo del plan, la cantidad aportada por UF como consecuencia de la compra de las distribuidoras es 107.3 millones, $32 \%$ del total.

La mayoría de las obras del PER no la realizan directamente por UF, sino que subcontrata las distintas actividades requeridas a otras empresas. Esta modalidad favoreció la llegada a Guatemala de otras compañías españolas vinculadas a UF. De acuerdo con la información suministrada por la embajada española, cinco empresas de este país especializadas en instalaciones eléctricas se establecieron en el país tras las inversiones realizadas por UF e Iberdrola. La competencia implicó un efecto de expulsión para pequeñas empresas locales que anteriormente realizaban estos mismos trabajos para el INDE.

Al margen de estas cuestiones, existe un alto grado de cumplimiento de los objetivos establecidos en el plan en cuanto al número de usuarios conectados. En la Tabla 10 se muestra la evolución de los indicadores de electrificación rural. Según los objetivos establecidos como consecuencia del desarrollo del PER, en 2004 se debería alcanzar un índice de $80 \%$, nivel que estaba ya próximo a conseguirse a finales de 2001. Desde que se firma el contrato de compraventa a mediados de 1999, hasta finales de 2001, se habían desembolsado 65.5 millones de US\$ por concepto de obras de distribución, lo cual permitió conectar 100657 nuevos clientes en 908 comunidades. Las inversiones en la infraestructura de transmisión hasta esa fecha ascendían a 40.2 millones de US\$.

Los resultados no son tan satisfactorios cuando lo que se analiza son las obras de transmisión realizadas. De acuerdo con el contenido del PER, el destino de los fondos del fideicomiso se desglosaba de la siguiente forma: obras de transmisión: 150.9 millones de US\$; electrificación rural: 182.7 millones de US\$.

Esto significa que las obras de extensión de la red de distribución (electrificación rural) deberían ir acompañadas de mejoras y ampliación de la red de transporte relativa a la extensión del área de distribución. Estas obras de transmisión resultan necesarias para soportar el aumento de la carga derivada de una expansión de la red de distribución y del incremento en el número de usuarios. Los indicadores de calidad expuestos en el apartado anterior mostraron la baja calidad del suministro de energía en las zonas rurales, motivado en gran parte por el retraso en la ejecución de las obras de transmisión. 
Tabla 11

Guatemala: indicadores de electrificación

\begin{tabular}{lccccc}
\hline & $\begin{array}{c}\text { Población } \\
(\text { miles })\end{array}$ & $\begin{array}{c}\text { Habitantes } \\
\text { por vivienda }\end{array}$ & $\begin{array}{c}\text { Total de } \\
\text { viviendas (miles) }\end{array}$ & $\begin{array}{c}\text { Clientes } \\
\text { residenciales (miles) }\end{array}$ & $\begin{array}{c}\text { Índice de } \\
\text { electrificación (\%) }\end{array}$ \\
\hline 1980 & 6917 & 5.7 & 1213.5 & 276.0 & 22.7 \\
1985 & 7575 & 5.8 & 1306.1 & 407.7 & 31.2 \\
1990 & 8749 & 5.9 & 1477.9 & 539.9 & 36.5 \\
1995 & 9976 & 6.0 & 1654.4 & 778.5 & 47.1 \\
1996 & 10243 & 6.1 & 1690.3 & 817.2 & 48.3 \\
1997 & 10517 & 6.1 & 1729.8 & 971.7 & 56.2 \\
1998 & 10799 & 6.1 & 1767.4 & 1079.7 & 61.1 \\
1999 & 11088 & 6.1 & 1808.9 & 1175.2 & 65.0 \\
2000 & 11385 & 6.1 & 1857.3 & 1328.1 & 71.5 \\
2001 & 11678 & 6.1 & 1905.1 & 1523.1 & 79.9 \\
\hline
\end{tabular}

Fuente: CEPAL, sobre la base de cifras oficiales.

En definitiva, el fideicomiso resulta ser un subsidio a la ET para desarrollar una actividad que de otra forma no resultaría rentable. Es decir, la privatización no ha alterado las características de una actividad que sigue siendo deficitaria y que, al menos en su fase inicial, requiere los recursos del Estado. En el medio plazo, la viabilidad y el sostenimiento de la electrificación rural puede debilitarse por los altos precios de la energía. Teniendo en cuenta que se habla de una población no acostumbrada al consumo eléctrico y de bajos ingresos, un elevado coste de la energía impedirá un crecimiento sostenido de su consumo.

En la actualidad existe un diferencial importante entre el consumo en las zonas urbanas (170kwh al mes) y en las rurales (50kwh al mes). Para la disminución de estas diferencias y la expansión del consumo en el área rural será fundamental el aumento de sus ingresos así como la evolución de los precios de la energía. Éstos tenderían a aumentar en 2003 con la finalización del contrato INDE-UF de suministro de energía. ${ }^{18}$

La realización por parte de UF de un PER ha permitido una rápida expansión del servicio. Dejando al margen posibles apreciaciones sobre el origen de los fondos, es evidente que el proceso de electrificación rural encontrará importantes dificultades en un futuro no muy lejano. Si no se da un aumento significativo de los ingresos en el área rural o se experimenta una reducción del precio de la energía, la sostenibilidad del proceso se debilitará gradualmente. Será difícil que esta actividad resulte rentable para las empresas distribuidoras por lo cual las inversiones en mejoras y ampliación de la red tenderán a estancarse. Ello

18 De ahí que la empresa esté desarrollando un proyecto de generación que le permita suministrar energía a sus propias distribuidoras a un precio menor que el que se maneja en el mercado. Aunque tradicionalmente se considera que la demanda de energía es inelástica, al menos en el corto y medio plazo, este nivel de inelasticidad depende del sector de consumo del que se esté hablando. No es lo mismo el sector industrial que el residencial y, dentro de este último, tampoco será igual la respuesta ante variaciones en los precios en función del nivel de ingresos de las familias. Cuanto menores sean los ingresos, más elástica será la demanda, incluso en el corto plazo. 
siempre y cuando el Estado no ponga en marcha nuevos mecanismos para subsidiar dicha actividad. Por tanto, es bastante probable que en ausencia de los mismos, la calidad en el suministro de electricidad a las zonas rurales no mejore o incluso empeore en el medio plazo.

\section{Balance final}

Desde la década de los ochenta, las empresas eléctricas comienzan a extender sus actividades al terreno internacional, favorecidas por la implantación de una serie de medidas de liberalización y privatización de los servicios públicos. En Guatemala este proceso se desarrolló, aunque con cierto retraso, de forma muy similar a la de otros países de América Latina. La principal diferencia con respecto a algunas de las grandes economías de esta región se encuentra en el reducido tamaño del mercado, cuestión que resulta fundamental para la concreción final de las estrategias y modalidades adoptadas. Dicho tamaño del mercado y la situación particular de la economía podían constituir factores desestimulantes para la entrada de inversión extranjera, por lo cual las autoridades desarrollaron mecanismos que compensaran estas debilidades. Así, la posibilidad de obtener determinados contratos de generación o la garantía de un PER financiado por el gobierno se han convertido en factores de localización fundamentales para las ET que han invertido en el país, inversiones que se han caracterizado, en la mayoría de los casos, por su carácter rentista. Estos factores de localización han dado lugar a resultados contradictorios.

Las principales aportaciones se dieron en aspectos tales como la mejora de los indicadores de garantía del suministro. Sin embargo, estos logros relativos estuvieron acompañados de otros efectos tales como la subida en el precio de las tarifas, el aumento en la dependencia de las importaciones de hidrocarburos y el mayor impacto ambiental derivado de la generación térmica a partir de derivados del petróleo. Además, el Estado ha tenido que subsidiar las tarifas y la energía comprada por las distribuidoras rurales como mecanismo para abaratar el costo de ésta, lo cual ha repercutido negativamente en el objetivo de control del gasto público. Por el lado de la calidad, los avances no han cubierto aún los compromisos adquiridos por las ET que compraron las distribuidoras públicas. En gran parte se debe al carácter poco estratégico de las inversiones en Guatemala y a la insuficiencia de las sanciones contempladas en las normas sobre calidad.

En cuanto a la electrificación rural, el acuerdo establecido entre el gobierno y uF como parte de la compra de las distribuidoras rurales permitió una expansión significativa de la cobertura en esta zona, lo cual fue posible gracias a la mediación del Estado quien, al igual que en tiempos pasados, está financiando la extensión de un servicio que no resulta rentable. La problemática en este sentido se derivará de la sostenibilidad de la situación. Dados los elevados precios de la energía, si el Estado no sigue subvencionando la distribución rural, la mayoría de la población no podrá hacer uso del servicio. La escasa rentabilidad 
proporcionada por este tipo de clientes a UF hacen preveer un empeoramiento de la calidad del servicio en ausencia de un mayor control y mejores medidas sancionadoras por parte del Estado.

En resumen, aunque algunos —no todos- de los objetivos planteados por las políticas sectoriales de atracción de capital extranjero se pueden considerar cumplidos, las estrategias utilizadas para ello han traído a su vez nuevos problemas para el sector. En otros casos, se agravaron algunos de los ya existentes. En definitiva, esto plantea la vulnerabilidad a mediano y largo plazos del actual modelo de desarrollo del sector eléctrico.

\section{Bibliografía}

AMM, Informe estadístico, varios años, Guatemala.

Altomonte, H., Las complejas mutaciones de la industria eléctrica de América Latina: falencias institucionales y regulatorias, CEPAL, División de Recursos Naturales e Infraestructura, Santiago de Chile, 2001.

Banco Mundial, World Development Indicators, Washington, 2001.

BCIE/BID/CEPAL, Sistema de interconexión eléctrica para los países de América Central. Hacia una integración regional de electricidad. El proyecto, oportunidades y desafíos, Madrid, 2001.

CEPAL, El mercado eléctrico regional: contratos PPAS en El Salvador, Guatemala, Nicaragua y Honduras, México, 2001a.

__ Evolución reciente y desafíos de los mercados mayoristas de electricidad en El Salvador, Guatemala y Panamá, México, 2001b.

$\longrightarrow$, Guatemala: informe sobre los contratos de compraventa de energía eléctrica suscritos por las empresas del estado en el período 1992-1997, versión preliminar, México, 2001c.

_- Istmo Centroamericano: la regulación de la distribución de energía eléctrica en los países con empresas privadas. Los casos de El Salvador, Nicaragua, Guatemala y Panamá, México, 2002.

- Istmo centroamericano: estadísticas del subsector eléctrico, México, varios años.

CNEE, Normas Técnicas del Servicio de Distribución, Resolución CNEE núm. 09-99, Guatemala, 1999. 2002 .
Coral, I., Ligazones eléctricas internacionales. Hacia una estrategia para propulsar su implantación, BID, Washington, DC, 2002.

EEGSA, Informe Anual 1999, Guatemala, 2000.

Fundación Solar/BID, Sustainability of Power Sector Reform in Latin America. The Reform in Guatemala, working paper, Wasington, DC, 2002.

Iberener, Memoria anual 2000, Madrid, 2001.

Instituto para la Diversificación y Ahorro de la Energía, Impactos ambientales de la producción eléctrica. Análisis de ciclo de vida de ocho tecnologías de generación eléctrica, Madrid, 2000.

IDC, La Energía eléctrica en Guatemala, Asociación General de Generadores, Guatemala, 1999.

INDE, El sector eléctrico en Guatemala, Gerencia de planificación, Guatemala, 1999.

IEA, Electricity information 2001, OECD/IEA, París, 2001.

Joskow, P. L., "Electricity sectors in transition", en The Energy Journal, vol. 19, núm. 2, Cambridge, 1998 , pp. $25-52$.

Lasheras, M. A., La regulación económica de los servicios públicos, Ariel Economía, Madrid, 1999.

Paz Antolín, M.J., "Los enfoques microeconómicos sobre la expansión de empresas transnacionales", en Boletín de Información Económica, núm. 2732, Ministerio de Economía y Hacienda, Madrid, 2002, pp. 37-44.

República de Guatemala, Ley General de Electricidad, Guatemala, 1996.

SYNEX, "Determinación de tarifas a nivel generación, transmisión y distribución en Guatemala", Informe de consultoría, Guatemala, 1997.

Unión Fenosa, Informe anual 2001, Madrid, 2002. 